\title{
Second-Order Duality for Continuous Programming Containing Support Functions
}

\author{
Iqbal Husain ${ }^{1}$, Mashoob Masoodi ${ }^{2}$ \\ ${ }^{1}$ Department of Mathematics, Jaypee University of Technology, Guna, (M.P), India \\ ${ }^{2}$ Department of Statistics, University of Kashmir, Hazratbal Srinagar, Kashmir, India \\ E-mail: ihusain11@yahoo.com,masoodisaba@yahoo.com \\ Received June 25, 2010; revised November 4, 2010; accepted November 9, 2010
}

\begin{abstract}
A second-order dual problem is formulated for a class of continuous programming problem in which both objective and constrained functions contain support functions, hence it is nondifferentiable. Under secondorder invexity and second-order pseudoinvexity, weak, strong and converse duality theorems are established for this pair of dual problems. Special cases are deduced and a pair of dual continuous problems with natural boundary values is constructed. A close relationship between duality results of our problems and those of the corresponding (static) nonlinear programming problem with support functions is briefly outlined.
\end{abstract}

Keywords: Continuous Programming, Second-Order Invexity, Second-Order Pseudoinvexity, Second-Order Duality, Nonlinear Programming, Support Functions, Natural Boundary Values

\section{Introduction}

Second-order duality in mathematical programming has been extensively investigated in the literature. A secondorder dual formulation for a non-linear programming problem was introduced by Mangasarian [1]. Later Mond [2] established various duality theorems under a condition which is called "Second order convexity". This condition is much simpler than that used by Mangasarian [1]. In [3], Mond and Weir reconstructed the second-order and higher order dual models to derive usual duality results. It is remarked here that second-order dual to a mathematical programming problem presents a tighter bound and because of which it enjoys computational advantage over a first order dual.

Duality and optimality for continuous programming have been widely investigated by many authors in the recent past, notably, Mond and Hanson [4], Bector, Chandra and Husain [5], Mond and Husain [6] and Chen [7] and other cited references in these expositions.

Chen [7] was the first to identify second-order dual formulated for a constrained variational problem and established various duality results under an involved invexity- like assumptions. Husain et al. [8] have presented Mond-Weir type duality for the problem of [7] and by introducing continuous-time version of second-order invexity and generalized second-order invexity, validated various duality results. Recently, Husain and Masoodi [9] have studied Wolfe type duality for a class of nondifferentiable continuous programming problem and established relationship between these results and the duality results of Husain et al. [10] for nonlinear programming problems with support functions.

In this paper, we formulate a Wolfe type second-order dual to a class of nondifferentiability continuous programming containing support functions. The popularity of this type of problems seems to originate from the fact that, even though the objective function and or/constraint functions are non-smooth, a simple representation of the dual problem may be found. The theory of non-smooth mathematical programming deals with more general type of functions by means of generalized sub-differentials. However, square root of positive semi-definite quadratic form and support functions are amongst few cases of the nondifferentiable functions for which one can write down the sub-or quasi-differentials explicitly. Here, various duality theorems for this pair of Wolfe type dual problems are validated under second-order invexity and second-order pseudoinvexity conditions. The special cases as in [1] are derived. A pair of Wolfe type dual variational problems with natural boundary values rather than fixed end points is presented and the proofs of its duality results are indicated. It is also shown that our second-order duality results can be considered as dynamic generalizations of corresponding (Static) secondorder duality results established for nonlinear program- 
ming problem with support function, considered by $\mathrm{Hu}-$ sain et al. [10].

\section{Pre-requisites and Expression of the Problem}

Let $I=[a, b]$ be a real interval, $\phi: I \times R^{n} \times R^{n} \rightarrow R$ and $\psi: I \times R^{n} \times R^{n} \rightarrow R^{m}$ be twice continuously differentiable functions. In order to consider $\phi(t, x(t), \dot{x}(t))$ where $x: I \rightarrow R^{n}$ is differentiable with derivative $\dot{x}$, denoted by $\phi_{x}$ and $\phi_{\dot{x}}$, the first order of $\phi$ with respect to $x(t)$ and $\dot{x}(t)$, respectively, that is,

$$
\phi_{x}=\left(\frac{\partial \phi}{\partial x^{1}}, \frac{\partial \phi}{\partial x^{2}}, \cdots, \frac{\partial \phi}{\partial x^{n}}\right)^{T}, \phi_{\dot{x}}=\left(\frac{\partial \phi}{\partial \dot{x}^{1}}, \frac{\partial \phi}{\partial \dot{x}^{2}}, \cdots, \frac{\partial \phi}{\partial \dot{x}^{n}}\right)^{T}
$$

Denote by $\phi_{x x}$ the Hessian matrix of $\phi$, and $\psi_{x}$ the $m \times n$ matrix respectively, that is, with respect to $x(t)$, that is, $\phi_{x x}=\left(\frac{\partial^{2} \phi}{\partial x^{i} \partial x^{j}}\right), i, j=1,2, \cdots, n, \psi_{x}$ the $m \times n$ matrix

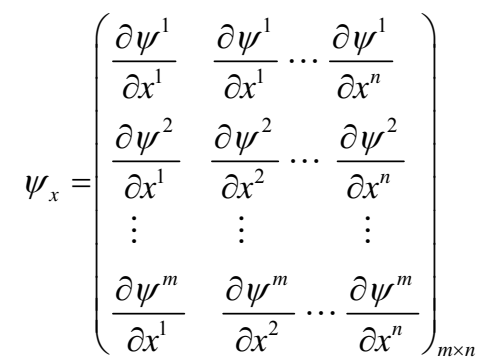

The symbols $\phi_{\dot{x}}, \phi_{\dot{x} x}, \phi_{x \dot{x}}$ and $\psi_{\dot{x}}$ have analogous representations.

Designate by $\mathrm{X}$ the space of piecewise smooth functions $x: I \rightarrow R^{n}$, with the norm $\|x\|=\|x\|_{\infty}+\|D x\|_{\infty}$, where the differentiation operator $D$ is given by $u=D x \Leftrightarrow x(t)=\int_{a}^{t} u(s) d s$,

Thus $\frac{d}{d t}=D$ except at discontinuities.

We incorporate the following definitions which are required in the subsequent analysis.

Definition 2.1 (Second-Order Invex): If there exists a vector function $\eta=\eta(t, x, \bar{x}) \in R^{n}$

where $\eta: I \times R^{n} \times R^{n} \rightarrow R^{n}$ and with $\eta=0$ at $t=a$ and $t$ $=b$, such that for a scalar function $\phi(t, x, \dot{x})$, the functional $\int_{I} \phi(t, x, \dot{x}) d t$ where $\phi: I \times R^{n} \times R^{n} \rightarrow R$ satisfies

$$
\begin{aligned}
& \int_{I} \phi(t, x, \dot{x}) d t-\int_{I}\left\{\phi(t, \bar{x}, \dot{\bar{x}})-\frac{1}{2} p^{T}(t) G p(t)\right\} d t \\
& \geq \int_{I}\left\{\eta^{T} \phi_{x}(t, \bar{x}, \dot{\bar{x}})+(D \eta)^{T} \phi_{\dot{x}}(t, \bar{x}, \dot{\bar{x}})+\eta^{T} G p(t)\right\} d t,
\end{aligned}
$$

then $\int_{I} \phi(t, x, \dot{x}) d t$ is second-order invex with respect to $\eta$ where $G=\phi_{x x}-2 D \phi_{x \dot{x}}+D^{2} \phi_{\dot{x} \dot{x}}$, and $p \in C\left(I, R^{n}\right)$, the space of $n$-dimensional continuous vector functions.

Definition 2.2 (Second-Order Pseudoinvex): The functional $\int_{I} \phi(t, x, \dot{x}) d t$ is said to be second-order pseudoinvex with respect to $\eta$ if

$$
\begin{aligned}
& \int_{I}\left\{\eta^{T} \phi_{x}+(D \eta)^{T} \phi_{\dot{x}}+\eta^{T} G p(t)\right\} d t \geq 0 \Rightarrow \\
& \int_{I} \phi(t, x, \dot{x}) d t \geq \int_{I}\left\{\phi(t, \bar{x}, \dot{\bar{x}})-\frac{1}{2} p^{T}(t) G p(t)\right\} d t .
\end{aligned}
$$

Definition 2.3 (Second-Order Quasi-Invex): The functional $\int_{I} \phi(t, x, \dot{x}) d t$ is to be second-order quasi-invex with respect to $\eta$ if

$$
\begin{aligned}
& \int_{I} \phi(t, x, \dot{x}) d t \leq \int_{I}\left\{\phi(t, \bar{x}, \dot{\bar{x}})-\frac{1}{2} p(t)^{T} G p(t)\right\} d t \\
& \Rightarrow \int_{I}\left\{\eta^{T} \phi_{x}+(D \eta)^{T} \phi_{\dot{x}}+\eta^{T} G(t) p(t)\right\} d t \leq 0 .
\end{aligned}
$$

Consider the following nondifferentiable continuous programming problem (CP) with support functions of Husain and Jabeen [11]:

(CP): Minimize $\int_{I}\{f(t, x, \dot{x})+S(x(t) \mid K)\} d t$

Subject to

$$
\begin{gathered}
x(a)=0=x(b) \\
g^{j}(t, x, \dot{x})+S\left(x(t) \mid C^{j}\right) \leq 0, j=1,2, \cdots, m, t \in I
\end{gathered}
$$

where $f$ and $g$ are continuously differentiable and each $\mathrm{C}^{j},(\mathrm{j}=1,2, \cdots, \mathrm{m})$ is a compact convex set in $\mathrm{R}^{\mathrm{n}}$. In [11], Husain and Zamrooda derived the following optimality conditions for the problem (CP):

Lemma 2.1 (Fritz-John Neccesary Optimality Conditions): If the problem (CP) attains a minimum at $x=\bar{X} \in X$, there exist $r \in R$ and piecewise smooth function $\bar{y}: I \rightarrow R^{m}$ with $\bar{y}(t)=\left(\bar{y}^{1}(t), \bar{y}^{2}(t), \cdots, \bar{y}^{m}(t)\right)$, $\bar{Z}: I \rightarrow R^{n}$ and $w^{j}: I \rightarrow R^{n}, j=1,2, \cdots, m$, such that

$$
\begin{gathered}
r\left[f_{x}(t, \bar{x}, \dot{\bar{x}})+\bar{z}(t)\right]+\sum_{j=1}^{m} \bar{y}^{j}(t)\left[g^{j}(t, \bar{x}, \dot{\bar{x}})+\bar{w}^{j}(t)\right] \\
=D\left[r f_{\dot{x}}(t, \bar{x}, \dot{\bar{x}})+\bar{y}(t)^{T} g_{\dot{x}}(t, \bar{x}, \dot{\bar{x}})\right], t \in I \\
\sum_{j=1}^{m} \bar{y}^{j}(t)\left[g^{j}(t, \bar{x}, \dot{\bar{x}})+\bar{x}(t)^{T} \bar{w}^{j}(t)\right]=0, t \in I \\
\bar{x}(t)^{T} \bar{z}(t)=S(\bar{x}(t) \mid K), t \in I \\
\bar{x}(t)^{T} \bar{w}^{j}(t)=S\left(\bar{x}(t) \mid C^{j}\right), j=1,2, \cdots, m, t \in I
\end{gathered}
$$




$$
\begin{gathered}
\bar{z}(t) \in K, w^{j}(t) \in C^{j}, j=1,2, \cdots, m, t \in I \\
(r, \bar{y}(t)) \geq 0, t \in I \\
(r, \bar{y}(t)) \neq 0, t \in I
\end{gathered}
$$

The minimum $\bar{x}$ of (CP) may be described as normal if $\bar{r}=1$ so that the Fritz John optimality conditions reduce to Karush-Kuhn-Tucker optimality conditions. It suffices for $\bar{r}=1$ that Slater's condition holds at $\bar{x}$.

Now we review some well known facts about a support function for easy reference.

Let $\Gamma$ be a compact set in $R^{n}$, then the support function of $\Gamma$ is defined by

$$
S(x(t) \mid \Gamma)=\max \left\{x(t)^{T} v(t): v(t) \in \Gamma, t \in I\right\}
$$

A support function, being convex everywhere finite, has a subdifferential in the sense of convex analysis i.e., there exist $z(t) \in R^{n}, t \in I$, such that

$$
S(y(t) \mid \Gamma)-S(x(t) \mid \Gamma) \geq(y(t)-x(t))^{T} z(t)
$$
by

From [12], the subdifferential of $S(x(t) \mid \Gamma)$ is given $\partial S(x(t) \mid \Gamma)=\left\{z(t) \in \Gamma, t \in I\right.$ such that $\left.\mid x(t)^{T} z(t)=S(x(t) \mid \Gamma)\right\}$.

For any set $A \subset R^{n}$, the normal cone to $\mathrm{A}$ at a point $x(t) \in A$ is defined by

$$
N_{A}(x(t))=\left\{y(t) \in R^{n} \mid y(t)(z(t)-x(t)) \leq 0, \forall z(t) \in A\right\}
$$

It can be verified that for a compact convex set $B$, $y(t) \in N_{B}(x(t))$ if and only if

$$
S(y(t) \mid B)=x(t)^{T} y(t), t \in I
$$

\section{Second Order Duality}

The following problem is formulated as Wolfe type dual for the problem (CP):

\section{(CD): Maximize}

$$
\int_{I}\left\{f(t, u, \dot{u})+\bar{u}(t)^{T} z(t)+\sum_{j=1}^{m} y^{j}(t)^{T}\left(g^{j}(t, u, \dot{u})+u(t)^{T} w^{j}(t)\right)\right.
$$

Subject to

$$
\left.-\frac{1}{2} p(t)^{T} H(t) p(t)\right\} d t
$$

$$
\begin{gathered}
u(a)=0=u(b) \\
f_{u}(t, u, \dot{u})+z(t)+\sum_{j=1}^{m} y^{j}(t)^{T}\left(g_{u}^{j}(t, u, \dot{u})+w^{j}(t)\right) \\
-D\left(f_{\dot{u}}(t, u, \dot{u})+y(t)^{T} g_{\dot{u}}(t, u, \dot{u})\right)+H(t) p(t)=0, t \in I \\
z(t) \in K, w^{j}(t) \in C^{j}, t \in I, j=1,2, \cdots, m \\
y(t) \geq 0, t \in I
\end{gathered}
$$

where

$$
\begin{aligned}
& H(t)=f_{u u}(t, u, \dot{u})+\left(y(t)^{T} g_{u}(t, u, \dot{u})\right)_{u}- \\
& 2 D\left[f_{u \dot{u}}(t, u, \dot{u})+\left(y(t)^{T} g_{u}(t, u, \dot{u})\right)_{\dot{u}}\right] \\
& +D^{2}\left[f_{u \dot{u}}(t, u, \dot{u})+\left(y(t)^{T} g_{\dot{u}}(t, u, \dot{u})\right)_{\dot{u}}\right]
\end{aligned}
$$

If $p(t)=0, t \in I$, the above dual becomes the dual of the problem studied in [11].

Theorem 3.1 (Weak duality): Let $x(t) \in X$ be a feasible solution of (CP) and $u(t), y(t), z(t), w^{1}(t), \quad\left(w^{2}(t), \ldots, w^{m}(t), p(t)\right) \quad$ be feasible solution for (CD). If for all feasible

$$
\left(x(t), u(t), y(t), z(t), w^{1}(t), w^{2}(t), \cdots, w^{m}(t), p(t)\right)
$$

and with respect to $\eta=\eta(t, x, u)$

(i) $\int_{I}\left\{f(t, . .)+.(\cdot)^{T} z(t)\right\} d t$ and $\sum_{j=1}^{m} \int_{I}\left\{y^{j}(t)\left(g^{j}(t, . .)+.(.) w^{j}(t)\right)\right\} d t$ second-order invex .

$\mathrm{Or}$

$$
\text { (ii) } \int_{I}\left\{f(t, . . .)+(.)^{T} z(t)+\sum_{j=1}^{m} y^{j}(t)^{T}\left(g^{j}(t, . . .)+(.) w^{j}(t)\right)\right\} d t
$$
is second-order pseudoinvex then. $\inf (\mathrm{CP}) \geq \sup (\mathrm{CD})$.

\section{Proof:}

$$
\begin{aligned}
& \int_{I}\{f(t, x, \dot{x})+S(x(t) \mid K)\} d t-\int_{I}\left\{f(t, u, \dot{u})+u(t)^{T} z(t)+\sum_{j=1}^{m} y^{j}(t)^{T}\left(g^{j}(t, u, \dot{u})+u(t)^{T} w^{j}(t)\right)-\frac{1}{2} p(t)^{T} H(t) p(t)\right\} d t \\
\geq & \int_{I}\left\{f(t, x, \dot{x})+x(t)^{T} z(t)\right\} d t-\int_{I}\left\{f(t, u, \dot{u})+u(t)^{T} z(t)\right\} d t-\sum_{j=1}^{m} \int_{I} y^{j}(t)\left(g^{j}(t, u, \dot{u})+u(t)^{T} w^{j}(t)\right) d t+\int_{I} \frac{1}{2} p(t)^{T} H(t) p(t) d t \\
\geq & \int_{I}\left[\eta^{T}\left\{f_{u}(t, u, \dot{u})+z(t)++(D \eta)^{T} f_{\dot{u}}(t, u, \dot{u})+\eta^{T} F(t) p(t)\right\} d t\right]-\int_{I} p(t)^{T} F(t) p(t) d t-\sum_{j=1}^{m} \int_{I} y^{j}(t)^{T}\left(g^{j}(t, u, \dot{u})+u(t)^{T} w^{j}(t)\right) d t \\
+ & \int_{I} \frac{1}{2} p(t)^{T} H(t) p(t) d t,
\end{aligned}
$$


(using $F(t)=f_{x x}-2 D f_{x \dot{x}}+D^{2} f_{\dot{x} \dot{x}}$ and the second-order

$\left.\int_{I}\left\{f(t, . .)+.(.)^{T} z(t)\right\} d t\right)$

$=\int_{I}\left[\eta^{T}\left\{f_{u}(t, u, \dot{u})+z(t)++D f_{\dot{u}}(t, u, \dot{u})+F(t) p(t)\right\} d t\right]+\left.\eta^{T} f_{\dot{u}}(t, u, \dot{u})\right|_{t=a} ^{t=b}-\sum_{j=1}^{m} \int_{I} y^{j}(t)^{T}\left(g^{j}(t, u, \dot{u})+u(t)^{T} w^{j}(t)\right) d t$

$-\int_{I} \frac{1}{2} p(t)^{T} F(t) p(t) d t+\int_{I} \frac{1}{2} p(t)^{T} H(t) p(t) d t$

$=-\int_{I} \eta^{T}\left[\sum_{j=1}^{m} \int_{I} y^{j}(t)^{T}\left(g_{\dot{u}}^{j}(t, u, \dot{u})+w^{j}(t)\right)-D\left(y(t)^{T} g_{\dot{u}}(t, u, \dot{u})\right)-G(t) p(t)\right] d t-\sum_{j=1}^{m} \int_{I} y^{j}(t)^{T}\left(g^{j}(t, u, \dot{u})+u(t)^{T} w^{j}(t)\right) d t$ $-\int_{I} \frac{1}{2} p(t)^{T} F(t) p(t) d t+\int_{I} \frac{1}{2} p(t)^{T} H(t) p(t) d t$

$=-\int_{I} \eta^{T}\left[\sum_{j=1}^{m} \int_{I} y^{j}(t)^{T}\left(g_{\dot{u}}^{j}(t, u, \dot{u})+w^{j}(t)\right)+(D \eta)^{T} y(t)^{T} g_{\dot{u}}+G(t) p(t) G(t) p(t)\right] d t-\sum_{j=1}^{m} \int_{I} y^{j}(t)^{T}\left(g^{j}(t, u, \dot{u})+u(t)^{T} w^{j}(t)\right) d t$ $-\int_{I} \frac{1}{2} p(t)^{T} F(t) p(t) d t+\int_{I} \frac{1}{2} p(t)^{T} H(t) p(t) d t$

$\geq-\sum_{j=1}^{m} \int_{I} y^{j}(t)^{T}\left(g^{j}(t, x, \dot{x})+u(t)^{T} w^{j}(t)\right) d t-\int_{I} \frac{1}{2} p(t)^{T} G(t) p(t) d t-\int_{I} \frac{1}{2} p(t)^{T} F(t) p(t) d t+\int_{I} \frac{1}{2} p(t)^{T} H(t) p(t) d t$

$\geq-\sum_{j=1}^{m} \int_{I} y^{j}(t)^{T}\left(g^{j}(t, x, \dot{x})+S\left(x(t) \mid C^{j}\right)\right) d t \geq 0$

This implies,

$\int_{I}(f(t, x, \dot{x})+S(x(t) \mid K)) d t \geq \int_{I}\left\{f(t, u, \dot{u})+u(t)^{T} z(t)+\sum_{j=1}^{m} y^{j}(t)\left(g^{j}(t, x, \dot{x})+u(t)^{T} w^{j}(t)\right)-\frac{1}{2} p(t)^{T} H(t) p(t)\right\} d t$ yielding,

$\inf (\mathrm{CP}) \geq \sup (\mathrm{CD})$.

$$
\begin{aligned}
& 0=\int_{I}\left[\eta ^ { T } \left\{f_{u}(t, u, \dot{u})\right.\right.\left.\left.+z(t)+\sum_{j=1}^{m} y^{j}(t)^{T}\left(g^{j}(t, u, \dot{u})+u(t)^{T} w^{j}(t)\right)-D\left(f_{\dot{u}}(t, u, \dot{u})+y(t)^{T} g_{\dot{u}}(t, u, \dot{u})\right)+H(t) p(t)\right\} d t\right] \\
&= \int_{I}\left[\eta ^ { T } \left\{f_{u}(t, u, \dot{u})+z(t)+\sum_{j=1}^{m} y^{j}(t)^{T}\left(g_{u}^{j}(t, u, \dot{u})+w^{j}(t)\right)+\right.\right. \\
&\left.\left.(D \eta)^{T}\left(f_{\dot{u}}(t, u, \dot{u})+y(t)^{T} g_{\dot{u}}(t, u, \dot{u})\right)+\eta^{T} H(t) p(t)\right\} d t-\left.\eta^{T}\left(f_{\dot{u}}+y g_{\dot{u}}\right)\right|_{t=a} ^{t=b}\right]
\end{aligned}
$$

(by integrating by parts)

Using boundary conditions (2.1) and (3.1), we have

$$
\int_{I}\left[\eta^{T}\left\{f_{u}(t, u, \dot{u})+z(t)+\sum_{j=1}^{m} y^{j}(t)\left(g_{u}^{j}(t, u, \dot{u})+w^{j}(t)\right)\right\}+(D \eta)^{T}\left(f_{\dot{u}}(t, u, \dot{u})+y(t)^{T} g_{\dot{u}}(t, u, \dot{u})\right)+\eta^{T} H(t) p(t)\right] d t=0
$$

This, in view of second-order pseudo-invexity of yields,

$$
\begin{aligned}
\int_{I}\left\{f(t, ., .)+(\cdot)^{T} z(t)+\sum_{j=1}^{m} y^{j}(t)^{T}\left(g^{j}(t, \cdot, \cdot)+(\cdot) w^{j}(t)\right)\right\} d t \\
\quad \int_{I}\left\{f(t, x, \dot{x})+x(t)^{T} z(t)+\sum_{j=1}^{m} y^{j}(t)\left(g^{j}(t, x, \dot{x})+x(t)^{T} w^{j}(t)\right)\right\} d t \\
\geq \int_{I}\left\{f(t, u, \dot{u})+u(t)^{T} z(t)+\sum_{j=1}^{m} y^{j}(t)\left(g^{j}(t, u, \dot{u})+u(t)^{T} w^{j}(t)\right)-\frac{1}{2} p(t)^{T} H(t) p(t)\right\} d t
\end{aligned}
$$




$$
\begin{aligned}
& \Rightarrow \int_{I}\left\{f(t, x, \dot{x})+S(x(t) \mid K)+\sum_{j=1}^{m} y^{j}(t)\left(g^{j}(t, x, \dot{x})+S\left(x(t) \mid C^{j}\right)\right)\right\} d t \\
& \geq \int_{I}\left\{f(t, u, \dot{u})+u(t)^{T} z(t)+\sum_{j=1}^{m} y^{j}(t)^{T}\left(g^{j}(t, u, \dot{u})+u(t)^{T} w^{j}(t)\right)-\frac{1}{2} p(t)^{T} H(t) p(t)\right\} d t
\end{aligned}
$$

Using (2.2) and (3.4) together with

$$
x(t)^{T} z(t) \leq S(x(t) \mid K)
$$

$$
\begin{aligned}
& \int_{I}\{f(t, x, \dot{x})+S(x(t) \mid K)\} d t \\
& \geq \int_{I}\left\{f(t, u, \dot{u})+u(t)^{T} z(t)+\sum_{j=1}^{m} y^{j}(t)\left(g^{j}(t, u, \dot{u})+u(t)^{T} w^{j}(t)\right)-\frac{1}{2} p(t)^{T} H(t) p(t)\right\} d t
\end{aligned}
$$

That is, inf (CP) $\geq \sup (\mathrm{CD})$.

Theorem 3.2 (Strong Duality): If $\bar{x}(t) \in X$ is a local (or global) optimal solution of (CP) and is also normal, then there exist piece wise smooth factor $y: I \rightarrow R^{m}$, $z: I \rightarrow R^{n}$ and $w^{j}: I \rightarrow R^{n}(j=1,2, \cdots, m)$ such that $\left(\bar{x}(t), \bar{y}(t), \bar{z}(t), \bar{w}^{1}(t), \bar{w}^{2}(t), \cdots, \bar{w}^{m}(t), p(t)=0\right)$ is a feasible solution of (CD) and the two objective values are equal. Furthermore, If the hypotheses of Theorem 3.1 hold, the $\left(\bar{x}(t), \bar{y}(t), \bar{z}(t), \bar{w}^{1}(t), \bar{w}^{2}(t), \cdots, \bar{w}^{m}(t), p(t)\right)$ is an optimal solution of (CD).

Proof: From Lemma 2.1, there exist piecewise smooth functions $y: I \rightarrow R^{m}, \mathrm{z}: I \rightarrow R^{n}$ and $w^{j}: I \rightarrow R^{n}(j=1,2, \cdots, m)$ satisfying

$$
\begin{aligned}
& f_{x}(t, \bar{x}, \dot{\bar{x}})+\bar{z}(t)+\sum_{j=i}^{m} \bar{y}^{j}(t)^{T}\left(g_{x}^{j}(t, \bar{x}, \dot{\bar{x}})+w^{j}\right) \\
& -D\left(f_{\dot{x}}(t, \bar{x}, \dot{\bar{x}})+\bar{y}(t)^{T} g_{\dot{x}}(t, \bar{x}, \dot{\bar{x}})\right)=0, t \in I
\end{aligned}
$$

$$
\begin{gathered}
f_{x}(t, \bar{x}, \dot{\bar{x}})+\bar{z}(t)+\sum_{j=i}^{m} \bar{y}^{j}(t)^{T}\left(g_{x}^{j}(t, \bar{x}, \dot{\bar{x}})+w^{j}\right) \\
-D\left(f_{\dot{x}}(t, \bar{x}, \dot{\bar{x}})+\bar{y}(t)^{T} g_{\dot{x}}(t, \bar{x}, \dot{\bar{x}})\right)=0, t \in I \\
\sum_{j=i}^{m} \bar{y}^{j}(t)^{T}\left(g_{x}^{j}(t, \bar{x}, \dot{\bar{x}})+w^{j}\right)=0, t \in I \\
\bar{x}(t)^{T} \bar{z}(t)=S(\bar{x}(t) \mid K), t \in I \\
\bar{x}(t)^{T} \bar{w}^{j}(t)=S\left(\bar{x}(t) \mid C^{j}\right), j=1,2, \cdots, m, t \in I \\
\bar{z}(t) \in K, w^{j}(t) \in C^{j}, j=1,2, \cdots, m, t \in I \\
\bar{y}(t) \geq 0, t \in I
\end{gathered}
$$

Hence

$\left(\bar{x}(t), \bar{y}(t), \bar{z}(t), \bar{w}^{1}(t), \bar{w}^{2}(t), \cdots, \bar{w}^{m}(t), p(t)=0\right) \quad$ satisfies the constraints of (CD) and

$$
\begin{aligned}
& \int_{I}\left\{f(t, \bar{x}, \dot{\bar{x}})+\bar{u}(t)^{T} \bar{z}(t)+\sum_{j=1}^{m} y^{j}(t)^{T}\left(g^{j}(t, \bar{x}, \dot{\bar{x}})+\bar{x}(t)^{T} \bar{w}^{j}(t)\right)-\frac{1}{2} p(t)^{T} H(t) p(t)\right\} d t \\
& =\int_{I}\{f(t, \bar{x}, \dot{\bar{x}})+S(x(t) / K)\} d t
\end{aligned}
$$

That is, the objective values are equal. Furthermore,

for every feasible solution, we have

$$
\begin{aligned}
& \int_{I}\left\{f(t, \bar{x}, \dot{\bar{x}})+\bar{u}(t)^{T} \bar{z}(t)+\sum_{j=1}^{m} y^{j}(t)^{T}\left(g^{j}(t, \bar{x}, \dot{\bar{x}})+\bar{x}(t)^{T} \bar{w}^{j}(t)\right)-\frac{1}{2} p(t)^{T} H(t) p(t)\right\} d t \\
& \geq \int_{I}\left\{f(t, u, \dot{u})+u(t)^{T} z(t)+\sum_{j=1}^{m} y^{j}(t)^{T}\left(g^{j}(t, u, \dot{u})+u(t)^{T} w^{j}(t)\right)-\frac{1}{2} p(t)^{T} H(t) p(t)\right\} d t
\end{aligned}
$$

So, $\left(\bar{x}(t), \bar{y}(t), \bar{z}(t), \bar{w}^{1}(t), \bar{w}^{2}(t), \cdots, \bar{w}^{m}(t)\right)$ is optimal for the problem (CD).

Theorem 3.3 (Converse duality): Let $f$ and $g$ are thrice continuously differentiable and $\left(\bar{x}(t), \bar{y}(t), \bar{z}(t), \bar{w}^{1}(t), \bar{w}^{2}(t), \cdots, \bar{w}^{m}(t), p(t)\right)$ be an optimal solution of (CD). If the following conditions hold:

$\left(A_{1}\right)$ : The Hessian matrix $H(t)$ is non-singular, and

$\left(\mathrm{A}_{2}\right):\left(\psi(t)^{T} H(t) \psi(t)\right)_{x}-D\left(\psi(t)^{T} H(t) \psi(t)\right)_{\dot{x}}$ 
$+2 \psi(t) D(H(t) \psi(t))_{\dot{x}}=0, t \in I$

$$
\Rightarrow \psi(t)=0, \quad t \in I
$$

Then $\bar{x}(t)$ is feasible solution of (CP), and $\sum_{j=1}^{m} \bar{y}^{j}(t)\left(g^{j}(t, \bar{x}, \dot{\bar{x}})+\bar{x}(t)^{T} \bar{w}^{j}(t)\right)=0, \quad t \in I$. In addition, if the hypotheses in Theorem 3.1 hold, then $\bar{x}(t)$ is an optimal solution of the problem (CP).

Proof: Since

$$
\left(\bar{x}(t), \bar{y}(t), \bar{z}(t), \bar{w}^{1}(t), \bar{w}^{2}(t), \cdots, \bar{w}^{m}(t), p(t)\right)
$$

is an optimal solution for (CD), then there exist piece wise smooth $\theta: I \rightarrow R^{n}$ and $\mu: I \rightarrow R^{m}$ such that following Fritz John type optimality conditions [7] are satisfied:

$$
\begin{aligned}
& \tau\left[\left(f_{x}(t, \bar{x}, \dot{\bar{x}})+\bar{z}(t)+\sum_{j=1}^{m} \bar{y}^{j}(t)\left(g_{x}(t, \bar{x}, \dot{\bar{x}})+\bar{w}^{j}(t)\right)\right)-\frac{1}{2}\left(p(t)^{T} H(t) p(t)\right)_{x}\right. \\
& \left.-D\left(f_{\dot{x}}(t, \bar{x}, \dot{\bar{x}})+\bar{y}(t)^{T} g(t, \bar{x}, \dot{\bar{x}})-\frac{1}{2}\left(p(t)^{T} H(t) p(t)\right)_{\dot{x}}\right)\right]+\theta(t)^{T}\left\{f_{x x}(t, \bar{x}, \dot{\bar{x}})+\left(y(t)^{T} g_{x}\right)_{x}\right. \\
& -D\left(f_{\dot{x} x}(t, \bar{x}, \dot{\bar{x}})+\left(y(t)^{T} g_{\dot{x}}\right)_{x}\right)+(H(t) p(t))_{x}-D\left(f_{x \dot{x}}(t, \bar{x}, \dot{\bar{x}})+\left(y(t)^{T} g_{x}\right)_{\dot{x}}\right) \\
& \left.-D\left(f_{\dot{x} \dot{x}}(t, \bar{x}, \dot{\bar{x}})+\left(y(t)^{T} g_{\dot{x}}\right)_{\dot{x}}\right)+(H(t) p(t))_{\dot{x}}\right\}=0, \quad t \in I \\
& \tau\left(g^{j}(t, \bar{x}, \dot{\bar{x}})+\left(\bar{x}(t) \bar{w}^{j}(t)\right)+\frac{1}{2} p(t)^{T} g_{x x}^{j} p(t)\right)+\theta(t)^{T}\left(g_{x x}^{j}-2 D g_{x \dot{x}}^{j}+D^{2} g_{\dot{x} \dot{x}}^{j}\right) p(t)+\mu^{j}(t)=0, t \in I, I=1,2, \cdots, m(3.7) \\
& \left(f_{x}(t, \bar{x}, \dot{\bar{x}})+\bar{z}(t)+\sum_{j=1}^{m} \bar{y}^{j}(t)\left(g^{j}(t, \bar{x}, \dot{\bar{x}})+\bar{w}^{j}(t)\right)\right)-D\left(f_{\dot{x}}(t, \bar{x}, \dot{\bar{x}})+\bar{y}(t)^{T} g_{\dot{x}}(t, \bar{x}, \dot{\bar{x}})\right)+(H(t) \bar{p}(t))=0, \quad t \in I \\
& \tau \bar{X}(t)^{T}+\theta(t) \in N_{K}(z(t)) \\
& (\tau, \mu(t), \theta(t)) \neq 0 \quad t \in I \\
& (\theta(t)-\tau \bar{p}(t))^{T} H(t)=0, t \in I \\
& \bar{\mu}(t)^{T} \bar{y}(t)=0, \quad t \in I \\
& (\tau, \mu(t)) \geq 0, t \in I \\
& f_{x}+z(t)+\sum_{j=1}^{m} \bar{y}^{j}(t)\left(g_{x}^{j}+w^{j}(t)\right)-\frac{1}{2}\left(p(t)^{T} H(t) p(t)\right)_{x}-D\left(f_{\dot{x}}+\bar{y}(t)^{T} g_{\dot{x}}-\frac{1}{2}\left(\bar{p}(t)^{T} B(t) \bar{p}(t)\right)_{\dot{x}}\right) \\
& +\theta(t)^{T}\left\{f_{x x}+\left(y(t)^{T} g_{x}\right)_{x}-D\left(f_{\dot{x} x}+\left(y(t)^{T} g_{\dot{x}}\right)_{x}\right)+(H(t) p(t))_{x}\right. \\
& \left.-D\left(f_{x \dot{x}}+\left(y(t)^{T} g_{x}\right)_{\dot{x}}\right)-D\left(f_{\dot{x} \dot{x}}(t, \bar{x}, \dot{\bar{x}})+\left(y(t)^{T} g_{\dot{x}}\right)_{\dot{x}}\right)+(H(t) p(t))_{\dot{x}}\right\}=0, t \in I
\end{aligned}
$$

Using the expression of $\mathrm{H}(\mathrm{t})$ and (3.16), this gives

$$
\begin{aligned}
& \bar{p}(t)^{T} H(t) \bar{p}(t)+D\left(\bar{p}(t)^{T} H(t) \bar{p}(t)\right)_{\dot{x}} \\
& -2 \bar{p}(t)^{T} D(H(t) \bar{p}(t))_{\dot{x}}=0, t \in I
\end{aligned}
$$

This, in view of the hypothesis $\left(\mathrm{A}_{2}\right)$ implies, yields,

$$
\bar{p}(t)=0, t \in I
$$

The relations (3.9) and (3.10) imply $\bar{x}(t)^{T} \in N_{K}(z(t))$ and $\bar{x}(t)^{T} \in N_{C_{j}}\left(w^{j}(t)\right), j=1,2, \cdots, m$ which respectively yields, $\bar{x}(t)^{T} \bar{z}(t)=S(\bar{x}(t) \mid K)$, $t \in I$ and

$$
\bar{x}(t)^{T} \bar{w}^{j}(t)=S\left(\bar{x}(t) \mid C^{j}\right), j=1,2, \cdots, m, t \in I
$$

The relation (3.7) with $p(t)=0, t \in I$ and (3.12) gives

$$
\sum_{j=1}^{m} \bar{y}^{j}(t)\left(g^{j}(t, \bar{x}, \dot{\bar{x}})+\bar{x}(t) \bar{w}^{j}(t)\right)=0, t \in I
$$

The relation (3.7) with $p(t)=0, t \in I, \quad \mu^{j}(t) \geq 0$, 
$t \in I$ and $\bar{x}(t)^{T} \bar{z}(t)=S(\bar{x}(t) \mid K), t \in I$, yields

$g^{j}(t, \bar{x}, \dot{\bar{x}})+S\left(\bar{x}(t) \mid C^{j}\right) \leq 0, j=1,2, \cdots, m, t \in I$

That is, $\bar{x}$ is feasible to (CP).

$$
\begin{aligned}
& \int_{I}\left\{f(t, \bar{x}, \dot{\bar{x}})+\bar{x}(t)^{T} \bar{z}(t)+\sum_{j=1}^{m} y^{j}(t)^{T}\left(g^{j}(t, \bar{x}, \dot{\bar{x}})+\bar{x}(t)^{T} \bar{w}^{j}(t)\right)-\frac{1}{2} p(t)^{T} H(t) p(t)\right\} d t \\
& =\int_{I} f(t, \bar{x}, \dot{\bar{x}})+S(\bar{x}(t) \mid K) d t
\end{aligned}
$$

This, along with the hypotheses of Theorem 3.1, yields that $\bar{x}(t)$ is an optimal solution of (CP).

\section{Special Cases}

Let for $t \in I, B(t)$ positive semi-definite matrices and continuous on $\mathrm{I}$. Then

$$
\left(x(t)^{T} B(t) x(t)\right)^{1 / 2}=S(x(t) \mid K), t \in I
$$

where $K=\left\{B(t) z(t) \mid z(t)^{T} B(t) z(t) \leq 1, t \in I\right\}$
Now, in view of $\bar{x}(t)^{T} \bar{z}(t)=S(\bar{x}(t) \mid K), t \in I$ and (3.11) and

$$
\int_{I}\left\{f(t, u, \dot{u})+u(t)^{T} B(t) z(t)+y(t)^{T} g(t, u, \dot{u})-\frac{1}{2} p(t)^{T} H(t) p(t)\right\} d t
$$

Subject to

Replacing $S(x(t) \mid K)$ by $\left(x(t)^{T} B(t) x(t)\right)^{1 / 2}$ and suppressing each $S\left(x(t) \mid C^{j}\right), \mathrm{j}=1,2, \cdots, \mathrm{m}$ from the constraints of $(\mathrm{CD})$, we have following problems treated by Husain and Masoodi [9]

$\left(\mathbf{C P}_{2}\right)$ : Minimize $\int_{I}\left\{f(t, x, \dot{x})+\left(x(t)^{T} B(t) x(t)\right)^{1 / 2}\right\} d t$

Subject to

$$
\begin{gathered}
x(a)=0=x(b) \\
g(t, x, \dot{x}) \leq 0, t \in I
\end{gathered}
$$

(CD): Maximize

$$
\begin{gathered}
f(t, u, \dot{u})+u(t)^{T} B(t) z(t)+y(t)^{T} g_{u}(t, u, \dot{u})-D\left(f_{\dot{u}}(t, u, \dot{u})+y(t)^{T} g_{\dot{u}}(t, u, \dot{u})\right)+H(t) p(t)=0, t \in I \\
z(t)^{T} B(t) z(t) \leq 1, \quad t \in I \quad y(t) \geq 0 .
\end{gathered}
$$

\section{Problems with Natural Boundary Conditions}

In this section, we formulate a pair of nondifferentiable dual variational problems with natural boundary values rather than fixed end points. The proofs for duality theorems for this pair of dual problems is omitted as they follow immediately on the basis of analysis of the preceding section and, of course, slight modifications are needed on the lines of [12]. The problems are:

$\left(\mathbf{C P}_{\mathbf{0}}\right)$ : Minimize $\int_{I}\{f(t, x, \dot{x})+S(\bar{x}(t) \mid K)\} d t$

Subject to

$$
g(t, x, \dot{x})+S\left(\bar{x}(t) \mid C^{j}\right) \leq 0, t \in I, j=1,2, \cdots, m
$$

$\left(\mathbf{C D}_{0}\right)$ : Maximize

$$
\int_{I}\left\{f(t, x, \dot{x})+x(t)^{T} z(t)+\sum_{j=1}^{m} y^{j}(t)^{T}\left(g^{j}(t, x, \dot{x})+x(t)^{T} w^{j}(t)\right)\right.
$$

Subject to

$$
\left.-\frac{1}{2} p(t)^{T} H(t) p(t)\right\} d t
$$

$$
\begin{gathered}
f_{x}(t, x, \dot{x})+z(t)+\sum_{j=1}^{m} y^{j}(t)\left(g_{x}^{j}(t, x, \dot{x})+w^{j}(t)\right) \\
-D\left(f_{\dot{x}}(t, x, \dot{x})+y(t)^{T} g_{\dot{x}}(t, x, \dot{x})\right)+(H(t) \bar{p}(t))=0, t \in I \\
z(t) \in K, w^{j}(t) \in C^{j}, j=1,2, \cdots, m, t \in I \\
y(t) \geq 0, t \in I \\
f_{\dot{x}}(t, x, \dot{x})+\left.y(t)^{T} g_{\dot{x}}(t, x, \dot{x})\right|_{t=a}=0, \\
f_{\dot{x}}(t, x, \dot{x})+\left.y(t)^{T} g_{\dot{x}}(t, x, \dot{x})\right|_{t=b}=0,
\end{gathered}
$$

\section{Nonlinear Programming Problems}

If all functions in the problems $\left(\mathrm{CP}_{0}\right)$ and $\left(\mathrm{CD}_{0}\right)$ are independent of $t$, then these problems will reduce to the 
following nonlinear programming problems studied by Husain et al. [10].

$\left(\mathbf{C P}_{\mathbf{1}}\right)$ : Minimize $f(x)+S(\bar{x}(t) \mid K)$

Subject to

$$
g^{j}(x)+S\left(\bar{x}(t) \mid C^{j}\right) \leq 0, j=1,2, \cdots, m
$$

$\left(\mathbf{C D}_{1}\right)$ : Maximize

$$
f(u)+u^{T} z(t)+\sum_{j=1}^{m} y^{j}(t)^{T}\left(g^{j}(u)+u^{T} w^{j}(t)\right)-\frac{1}{2} p^{T} H p
$$

Subject to

$$
\begin{aligned}
& f(u)+z(t)+\sum_{j=1}^{m} y^{j}(t)^{T}\left(g_{u}^{j}(u)+w^{j}(t)\right)+H p=0^{\prime} \\
& z \in K, w^{j} \in C^{j}, j=1,2, \cdots, m .
\end{aligned}
$$

where $H=f_{u u}(u)+y^{T} g_{u u}(u)$.

\section{References}

[1] O. L. Mangasarian, "Second and Higher Order Duality in Non linear Programming," Journal of Mathematical Analysis and Applications, Vol. 51, 1979, pp. 605-620.

[2] B. Mond,"Second Order Duality in Non-Linear Programming," Opsearch, Vol. 11, 1974, pp. 90-99.

[3] B. Mond and T. Weir, "Generalized Convexity and Higher Order Duality," Journal of Mathematical Analysis and Applications, Vol. 46, 1974, pp. 169-174.

[4] B. Mond and M. A. Hanson, "Duality for Variational Problems," Journal of Mathematical Analysis and Applica- cations, Vol. 11, 1965, pp. 355-364.

[5] C. R. Bector, S. Chandra and I. Husain, "Generalized Concavity and Duality in Continuous Programming," Utilitas Mathematica, Vol. 25, 1984.

[6] B. Mond and I. Husain, "Sufficient Optimality Criteria and Duality for Avariational Problem with Generalized Invexity," The Journal of the Australian Mathematical Society. Series B. Applied Mathematics, Vol. 31, No. 1, 1989, pp. 101-121.

[7] X. Chen, "Second-Order Duality for the Variational Problems," Journal of Mathematical Analysis and Applications, Vol. 216, 2003, pp. 261-270.

[8] I. Husain, A. Ahmed and M. Masoodi, "Second Order Duality for Variational Problems," European Journal of Pure and Applied Mathematics, Vol. 2, No. 2, 2009, pp. 271- 295.

[9] I. Husain and M. Masoodi, "Second-Order Duality for a Class of Nondifferentiable Continuous Programming Problems," European Journal of Pure and Applied Mathematics, 2010, (In Press).

[10] I. Husain, A. Ahmed and M. Masoodi, "Second-Order Duality in Mathematical Programming with Support Functions," Journal of Informatics and Mathematical Sciences, Vol. 1, No. 2-3, 2009, pp. 165-182.

[11] I. Husain and Z. Jabeen, "Continuous Programming Containing Support Functions," Journal of Applied Mathematics and Informatics, Vol. 26, No. 1-2, 2008, pp. 75-106.

[12] B. Mond and M. Schechter, "Nondifferentiable Symmetric Duality," Bulletin of the Australian Mathematical Society, Vol. 53, 1996, pp. 177-188. 